\title{
ASYMPTOTICS FOR STIELTJES POLYNOMIALS, PADÉ-TYPE APPROXIMANTS, AND GAUSS-KRONROD QUADRATURE
}

\author{
M. BELLO HERNÁNDEZ, B. DE LA CALLE YSERN, J.J. GUADALUPE HERNÁNDEZ, \\ AND G. LÓPEZ LAGOMASINO
}

\begin{abstract}
We study the asymptotic properties of Stieltjes polynomials outside the support of the measure as well as the asymptotic behaviour of their zeros. These properties are used to estimate the rate of convergence of sequences of rational functions, whose poles are partially fixed, which approximate Markov-type functions. An estimate for the speed of convergence of Gauss-Kronrod quadrature formula in the case of analytic functions is also given.
\end{abstract}

\section{INTRODUCTION}

1.1. General Remarks. Let $\omega$ be a nonnegative function on the interval $[-1,1]$ with $\omega \in$ $L^{1}[-1,1]$. By $d x$, we denote Lebesgue's measure on $[-1,1]$. Let $\left\{p_{n}\right\}_{n \in \mathbb{N}}$ be the sequence of orthonormal polynomials with respect to the weight function $\omega$; that is, $p_{n}(z)=\kappa_{n} z^{n}+$ $\cdots, \kappa_{n}>0$, and

$$
\int_{-1}^{1} p_{m}(x) p_{k}(x) \omega(x) d x=\delta_{k m}
$$

It is well known and easy to verify that there exists a unique monic polynomial $S_{n}$ of degree $n$ which satisfies the orthogonality relations

$$
\int_{-1}^{1} x^{k} S_{n}(x) p_{n-1}(x) \omega(x) d x=0, \quad k=0,1, \ldots, n-1 .
$$

The polynomial $S_{n}$ is called the $n$th Stieltjes polynomial with respect to the weight function $\omega$. This class of polynomials $\left\{S_{n}\right\}$ was introduced by Stieltjes [22] for the Legendre weight $w \equiv 1$ in terms of the associated function of the second kind. For more information see remark after Lemma 2 below.

In the last two decades Stieltjes polynomials have attracted considerable attention. This interest has been motivated by their connection with Gauss-Kronrod quadrature formulas

$$
\int_{-1}^{1} f(x) w(x) d x=\sum_{k=1}^{n} \sigma_{k, n} f\left(x_{k, n}\right)+\sum_{k=1}^{n+1} \gamma_{k, n} f\left(y_{k, n}\right)+E_{n}(f),
$$

1991 Mathematics Subject Classification. Primary: 41A21, 42C05; Secondary: 30E10.

Key words and phrases. Orthogonal polynomials, Padé-type approximation, Stieltjes polynomials, Gauss-Kronrod quadrature.

The work of M. Bello and J.J. Guadalupe was partially supported by DGES under grant PB96-0120C03-02 and UR, AP-98/B12.

J. J. Guadalupe died in a road accident on April 1, 2000. We, the co-authors, will miss a great friend, and the spanish mathematical community his leadership and dedication to research.

The work of G. López was partially supported by Dirección General de Enseñanza Superior under grant PB 96-0120-C03-01 and by INTAS under grant 93-0219 EXT. 
where $\left\{x_{k, n}\right\}$ are the zeros of the orthogonal polynomial $p_{n}$. The nodes $\left\{y_{k, n}\right\}$ and weights $\left\{\sigma_{k, n}\right\},\left\{\gamma_{k, n}\right\}$ are chosen so as to maximize the degree of exactness of the formula in the space of polynomials. It is easy to see that if for a given weight, $E_{n}(f)=0$ for all polynomials of degree less than or equal to $3 n+1$, then the nodes $y_{k, n}$ must be the zeros of the Stieltjes polynomial $S_{n+1}$. The reciprocal statement is also true if the zeros of the Stieltjes polynomials $S_{n+1}$ happen to be simple and distinct from the zeros of $p_{n}$. In fact, there is equivalence between the construction of Stieltjes polynomials and GaussKronrod quadrature formulas if multiple nodes are allowed (for details, see Section 5). Kronrod [10] was the first to consider this type of formulas taking as nodes the zeros of Legendre polynomials and the zeros of the corresponding Stieltjes polynomials. For further references and surveys on this topic, see [9], [12], and [7].

From the point of view of quadrature processes it is important to know if the nodes are simple, their interlacing properties, and certainly if they are contained in the set where the function to be integrated is defined. Since $S_{n}$ is orthogonal with respect to a sign changing function, equations (2), in general, do not guarantee that the zeros of $S_{n}$ lie in $[-1,1]$, that they are simple and distinct from the zeros of $p_{n-1}$, or even that they are real. However, for the ultraspherical weight function $w_{\lambda}, w_{\lambda}(x)=\left(1-x^{2}\right)^{\lambda-1 / 2}, 0 \leq \lambda \leq 2$, Szegö proved in [24] that these properties hold for all $n$. Positivity of the coefficients appearing in the quadrature formula and interlacing properties of the zeros have also been studied for the ultraspherical weights $w_{\lambda}, 0 \leq \lambda \leq 1$, in [11] and [4], respectively. The same properties are analysed in [16] and [17] for weights of the type $\sqrt{1-x^{2}} w(x)$, where $\sqrt{1-x^{2}} w(x)$ is positive and twice continuously differentiable on $[-1,1]$. Estimates of the error in Gauss-Kronrod quadrature formulas have been given for classes of functions with different degree of smoothness. For the case of analytic functions, see [6] and [15]. In connection with Lagrange interpolation see also [8].

Thus, to some extent the study of Stieltjes polynomials has been marked so far by their applicability in Gauss-Kronrod quadrature. This has caused that research has focused on weights for which quadrature is meaningful for classes of functions as large as possible. We have shifted the attention to the Stieltjes polynomials themselves and to the study of their asymptotic properties regardless of their immediate use in quadrature. We aim to describe general classes of weights for which the corresponding Stieltjes polynomials have either $n$th root (weak), ratio, or strong asymptotic behaviour. Such results have direct application in the approximation of Markov functions by means of rational approximants with partially prescribed poles (Padé-type approximants in the terminology commonly used in recent years). Regarding such approximants, we refer to the papers [1]-[3] and the references therein. As a by-product of the results obtained in rational approximation, we give estimates of the rate of convergence of Gauss-Kronrod quadrature for functions which are analytic on a neighbourhood of the set of integration.

1.2. Definitions and Known Results. Let $\mu$ be a finite, positive, Borel measure on the real line $\mathbb{R}$ whose compact support $S(\mu)$ contains infinitely many points. Let $\mu^{\prime}=d \mu / d x$ be the Radon-Nykodym derivative of $\mu$ with respect to the Lebesgue measure $d x$. Whenever we find it more convenient, we adopt the differential notation for a measure. The $n$th Stieltjes polynomial with respect to $\mu$ is defined by (1) and (2) substituting $\omega(x) d x$ by $d \mu(x)$. That is, let $\left\{S_{n}\right\}_{n \in \mathbb{N}}$ be a sequence of polynomials such that for each $n \in \mathbb{N}, S_{n}$ is 
defined as the monic polynomial of degree at most $n$ verifying

$$
\int_{S(\mu)} x^{k} S_{n}(x) p_{n-1}(x) d \mu(x)=0, \quad k=0,1, \ldots, n-1,
$$

where $p_{n-1}=\kappa_{n-1} z^{n-1}+\cdots, \kappa_{n-1}>0$, is the $(n-1)$ th orthonormal polynomial with respect to the measure $\mu$. Finding $S_{n}$ reduces to solving a system of $n$ homogeneous equations on $n+1$ unknowns. Thus a non-trivial solution always exists. From the orthogonality relations satisfied by $S_{n}$ it is easy to conclude that $\operatorname{deg} S_{n}=n$. $S_{n}$ is called the $n$th Stieltjes polynomial with respect to the measure $d \mu$. Unless otherwise stated, the set of integration is $S(\mu)$ in which case it will not be indicated. We will refer to $s_{n}=\kappa_{n-1} S_{n}$ as the normalized $n$th Stieltjes polynomial. The introduction of this notation allows to give several formulas a closer form; of course, it has nothing to do with attempting to orthonormalize the Stieltjes polynomials.

The largest class of measures with which we will deal is that of regular measures. This class of measures was introduced in recent years and has been extensively studied. The excellent monography [21] by H. Stahl and V. Totik is dedicated to the study of these measures and their orthogonal polynomials. For the precise definition and different equivalent forms of its expression see page 61 of that treatise. The regularity of the measure $\mu$, which is denoted $\mu \in \mathbf{R e g}$, is equivalent to either one of the following two limit relations (see Theorem 3.1.1 in [21])

$$
\begin{gathered}
\lim _{n \rightarrow \infty} \kappa_{n}^{1 / n}=\frac{1}{\operatorname{cap} S(\mu)}, \\
\lim _{n \rightarrow \infty}\left|p_{n}(z)\right|^{1 / n}=\exp \left\{g_{\Omega}(z, \infty)\right\},
\end{gathered}
$$

uniformly on compact subsets of $\mathbb{C} \backslash \operatorname{Co}(S(\mu))$, where $\operatorname{Co}(S(\mu))$ denotes the convex hull of $S(\mu)$, cap $S(\mu)$ stands for the logarithmic capacity of $S(\mu)$, and $g_{\Omega}(z, \infty)$ is the (generalized) Green function with singularity at infinity relative to the region $\Omega=\overline{\mathbb{C}} \backslash S(\mu)$ (cf. Section 1.2 and Appendix A.5 in [21] for the definition). We will assume that cap $S(\mu)>0$ which is equivalent to the fact that $g_{\Omega}(z, \infty) \not \equiv+\infty$.

The Blumenthal-Nevai class of measures is also of importance in the theory of orthogonal polynomials and related subjects. Let

$$
x p_{n}(x)=a_{n+1} p_{n+1}+b_{n} p_{n}(x)+a_{n} p_{n-1}(x), \quad n \geq 1,
$$

be the recurrence relation satisfied by the sequence $\left\{p_{n}\right\}_{n \in \mathbb{N}}$ of orthonormal polynomials. We say that $\mu \in M(a, b)$ if $\lim _{n \rightarrow \infty} b_{n}=b$ and $\lim _{n \rightarrow \infty} a_{n}=a / 2$. In this case it is known that $S(\mu)=[b-a, b+a] \cup e$, where $e$ is at most a denumerable set whose only possible accumulation points are $b \pm a$. We will assume that $a \neq 0$ so that $[b-a, b+a]$ does not reduce to a point. In this case

$$
\lim _{n \rightarrow \infty} \frac{p_{n+1}(z)}{p_{n}(z)}=\Psi\left(\frac{z-b}{a}\right)
$$

uniformly on compact subsets of $\mathbb{C} \backslash S(\mu)$, where $\Psi(z)=z+\sqrt{z^{2}-1}$. The square root is taken to be positive for $z>1$. This function is the conformal mapping of $\overline{\mathbb{C}} \backslash[-1,1]$ onto $\{|w|>1\}$ such that $\Psi(\infty)=\infty$ and $\Psi^{\prime}(\infty)>0$. Because of these properties, $\log |\Psi((z-b) / a)|$ happens to be the Green function with singularity at infinity relative to the region $\mathbb{C} \backslash[b-a, b+a]$. If $\mu \in M(a, b)$, in addition to (6), we have that for every 
bounded Borel-measurable function $f$ on $S(\mu)$, continuous on $[b-a, b+a]$, we have

$$
\lim _{n \rightarrow \infty} \int_{S(\mu)} f(x) p_{n}^{2}(x) d \mu(x)=\frac{1}{\pi} \int_{b-a}^{b+a} f(x) \frac{d x}{\sqrt{a^{2}-(x-b)^{2}}} .
$$

For more details on this class of measures and its properties see the book [13] by P. Nevai. A well known sufficient condition for $\mu \in M(a, b)$ due to E. A. Rakhmanov is that $S(\mu)=[b-a, b+a]$ and $\mu^{\prime}>0$ almost everywhere on $[b-a, b+a]$ (for a proof see, for example, [19]).

Finally, we consider the Szegő class of measures. For simplicity in the notation, we restrict our attention here to measures supported on $[-1,1]$. We say that $\mu \in \mathbf{S}$ if $S(\mu)=$ $[-1,1]$ and $\log \mu^{\prime}(x) / \sqrt{1-x^{2}} \in L^{1}[-1,1]$. In this case

$$
\lim _{n \rightarrow \infty} \frac{p_{n}(z)}{[\Psi(z)]^{n}}=\frac{1}{\sqrt{2 \pi}} S_{\mu}(\Psi(z))
$$

uniformly on compact subsets of $\overline{\mathbb{C}} \backslash[-1,1]$, where

$$
S_{\mu}(z)=\exp \left\{\frac{1}{4 \pi} \int_{0}^{2 \pi} \log \left(\mu^{\prime}(\cos \theta)|\sin (\theta)|\right) \frac{e^{i \theta}+z}{e^{i \theta}-z} d \theta\right\}, \quad|z| \neq 1 .
$$

Relations of type (8) are called exterior strong asymptotic formulas.

As for strong asymptotics on the support of the measure, it is necessary to place more restrictions on the measure $\mu$ to obtain some results. Thus, for instance, suppose that $d \mu(x)=w(x) d x$ and the function $f(\theta)=w(\cos \theta)|\sin \theta|$ satisfies the Lipschitz-Dini condition

$$
|f(\theta+\delta)-f(\theta)|<M(\log \delta)^{-L-1}
$$

where $M$ and $L$ are fixed positive numbers. Then, we have (see Theorem 12.1.4 in [23]) uniformly on $-1 \leq x \leq 1$

$$
\left(1-x^{2}\right)^{1 / 4} \sqrt{w(x)} p_{n}(x)=\sqrt{2 / \pi} \cos \{n \theta+\gamma(\theta)\}+\mathcal{O}\left\{(\log n)^{-L}\right\},
$$

where $x=\cos \theta, \exp \{i \gamma(\theta)\}=S_{\mu}\left(e^{i \theta}\right) /\left|S_{\mu}\left(e^{i \theta}\right)\right|$, and $S_{\mu}\left(e^{i \theta}\right):=\lim _{r \rightarrow 1^{-}} S_{\mu}\left(r e^{i \theta}\right)$.

1.3. Statement of Main Results. As mentioned above, the main object of this paper is the study of the asymptotic behaviour of Stieltjes polynomials. In this respect not much is known so far. Most of the results to the present are formulas of type (9), since they allow to obtain pretty accurate information on the location and asymptotic distribution of the zeros of Stieltjes polynomials. Ehrich, [4] and [5], proves relations similar to (9) for Stieltjes polynomials with respect to the ultraspherical weights $w_{\lambda}, 0 \leq \lambda \leq 2$. Previously, Peherstorfer had given in [16] a representation of the limit of the Stieltjes polynomials with respect to the weight $\sqrt{1-x^{2}} w(x)$ in terms of the series expansion of $S_{w}$ at $z=0$ provided that $\sqrt{1-x^{2}} w(x)$, is positive and twice continuously differentiable on $[-1,1]$ (see also [17]). It is quite surprising, that formulas for the exterior asymptotic behaviour of Stieltjes polynomials are only known for the class of weights considered in [17]. In that work, the author asks whether such a relation takes place under weaker assumptions. As we shall see, the only restriction is the least one possible; that is, that the measure satisfy Szegö's condition $(\mu \in \mathbf{S})$. Regarding other types of asymptotics as $n$th root or ratio asymptotics, to the best of our knowledge the results we present are the first available. 
Set

$$
E=\left\{z \in \mathbb{C}: g_{\Omega}(z, \infty) \leq \max _{w \in \operatorname{Co}(S(\mu))} g_{\Omega}(w, \infty)\right\} .
$$

We will write $S(\mu)=\operatorname{ess}[b-a, b+a]$ if the support of the measure $\mu$ has the same structure as in the Blumenthal-Nevai class $M(a, b)$; that is, consists of the interval $[b-a, b+a]$ and at most a denumerable set which may only accumulate at the points $b \pm a$.

The functions of second kind with respect to $\mu$ are given by

$$
g_{n}(z)=\int \frac{p_{n}(x)}{z-x} d \mu(x), \quad z \in \Omega=\overline{\mathbb{C}} \backslash S(\mu) .
$$

These functions are analytic in $\Omega$ and $g_{n}(\infty)=0$. Because of the orthogonality relations satisfied by $p_{n}$ with respect to the measure $\mu, z=\infty$ is a zero of $g_{n}$ of multiplicity $n+1$.

We have

Theorem 1. The following assertions hold:

a) If $\mu \in \mathbf{R e g}$ and $\operatorname{cap} S(\mu)>0$, then

$$
\lim _{n \rightarrow \infty} s_{n+1}(z) g_{n}(z)=1 \text {, }
$$

uniformly on compact subsets of $\overline{\mathbb{C}} \backslash E$. In addition, the set of accumulation points of the zeros of $\left\{S_{n+1}\right\}_{n \in \mathbb{N}}$ is contained in $E$ and

$$
\lim _{n \rightarrow \infty}\left|s_{n+1}(z)\right|^{1 / n}=\exp \left\{g_{\Omega}(z, \infty)\right\}
$$

uniformly on each compact subset of $\mathbb{C} \backslash E$. Moreover, if $S(\mu)=\operatorname{ess}[b-a, b+a]$ then relations (10) and (11) take place uniformly on each compact subsets of $\overline{\mathbb{C}} \backslash S(\mu)$ and $\mathbb{C} \backslash S(\mu)$ respectively, and the set of accumulation points of the zeros of the $\left\{S_{n}\right\}_{n \in \mathbb{N}}$ is contained in $S(\mu)$.

b) If $\mu \in M(a, b)$ with $a \neq 0$, then

$$
\lim _{n \rightarrow \infty} \frac{s_{n+1}(z)}{s_{n}(z)}=\Psi\left(\frac{z-b}{a}\right) \quad \text { and } \quad \lim _{n \rightarrow \infty} \frac{p_{n}(z)}{s_{n+1}(z)}=\frac{1}{\sqrt{(z-b)^{2}-a^{2}}},
$$

uniformly on each compact subset of $\mathbb{C} \backslash S(\mu)$.

c) If $\mu \in \mathbf{S}$, then

$$
\lim _{n \rightarrow \infty} \frac{s_{n+1}(z)}{[\Psi(z)]^{n}}=\sqrt{\frac{z^{2}-1}{2 \pi}} S_{\mu}(\Psi(z)),
$$

uniformly on each compact subset of $\mathbb{C} \backslash[-1,1]$, where $S_{\mu}(z)$ is as in (8).

The paper is organized as follows. The next section is essentially dedicated to prove an integral relation, between Stieltjes polynomials and functions of second kind, which plays an important role in the subsequent arguments. The following section is dedicated to the study of the asymptotic properties of Stieltjes polynomials and proving our main results stated above. In Section 4 , we apply our result on $n$th root asymptotics to obtain convergence of a certain type of Padé type approximants to Markov functions. In turn, this result is applied in Section 5 to estimate the speed of convergence of the Gauss-Kronrod quadrature formula when integrating functions which are analytic on a neighbourhood of the support of the measure. The final section contains an example illustrating the type of difficulties one encounters with the location of the zeros and the asymptotic properties of the Stieltjes polynomials when the support of the measure contains more than one interval. 


\section{Some Lemmas}

The next lemma provides some useful relations

Lemma 1. We have

$$
g_{n}(z) p_{n}(z)=\int \frac{p_{n}^{2}(x)}{z-x} d \mu(x), \quad z \in \Omega
$$

and for any polynomial $\ell_{n}$ of degree less than or equal to $n$

$$
\ell_{n}(z) \int \frac{s_{n}(x)}{z-x} p_{n-1}(x) d \mu(x)=\int \frac{\ell_{n}(x) s_{n}(x)}{z-x} p_{n-1}(x) d \mu(x), \quad z \in \Omega .
$$

Let $K$ be a compact subset of $\mathbb{C} \backslash \operatorname{Co}(S(\mu))$, then there exist positive constants $M_{1}, M_{2}$, independent of $n$, satisfying

$$
M_{1} \leq\left|g_{n}(z) p_{n}(z)\right| \leq M_{2}, \quad z \in K
$$

In particular, $p_{n} g_{n}$ has no zeros on $\mathbb{C} \backslash \operatorname{Co}(S(\mu))$. Moreover, if $S(\mu)=\operatorname{ess}[b-a, b+a]$, $a<a^{\prime}$, and $K$ is a compact subset of $\mathbb{C} \backslash\left(S(\mu) \cup\left[b-a^{\prime}, b+a^{\prime}\right]\right)$, then there exists $n_{0}$ such that for all $n \geq n_{0}, g_{n}$ has no zeros on $\mathbb{C} \backslash\left[b-a^{\prime}, b+a^{\prime}\right]$ and (16) takes place uniformly on $K$.

Proof. Relation (14) is well known (see e.g. Theorem 6.1 .8 in [21]). It follows directly from the orthogonality properties of $p_{n}$. To prove (15) notice that from the orthogonality relations satisfied by $s_{n}$ we have that

$$
\int \frac{\ell_{n}(z)-\ell_{n}(x)}{z-x} s_{n}(x) p_{n-1}(x) d \mu(x)=0,
$$

which is equivalent to (15).

The general statement concerning (16) is also well known. It is an immediate consequence of (14). The upper bound is quite obvious. For the lower bound, notice that

$$
g_{n}(z) p_{n}(z)=\int \frac{(\Re(z)-x) p_{n}^{2}(x)}{|z-x|^{2}} d \mu(x)+i \Im(z) \int \frac{p_{n}^{2}(x)}{|z-x|^{2}} d \mu(x), \quad z \in \Omega,
$$

which in the first place makes it obvious that $g_{n} p_{n}$ has no zero in $\mathbb{C} \backslash \operatorname{Co}(S(\mu))$, and secondly it is easy to bound from below in absolute value by a positive constant on a compact subset of $\mathbb{C} \backslash \operatorname{Co}(S(\mu))$.

Now, assume that $S(\mu)=\operatorname{ess}[b-a, b+a]$, and $\left[b-a^{\prime}, b+a^{\prime}\right]$ and $K$ are as in the second part of the statement relative to (16). In this case, the set $\operatorname{Co}(S(\mu)) \backslash\left(S(\mu) \cup\left[b-a^{\prime}, b+a^{\prime}\right]\right)$ is made up of at most a finite number of non-intersecting open intervals (let us assume that at least one, otherwise we would have nothing to prove). It is easy to prove that on the closure of any bounded connected component of $\mathbb{R} \backslash S(\mu), p_{n}$ can have at most one zero (if this were not so, one can construct a polynomial $\ell$ of degree $\leq n-2$ such that $\ell p_{n}$ has a constant sign on the support of the measure contradicting the orthogonality relations satisfied by $p_{n}$ ). On the other hand, each mass point of $\mu$ attracts at least one zero of $p_{n}$ (see Theorem 6.1.1 [23]). Therefore, for all sufficiently large $n$, each one of the nonintersecting open interval which compose $\operatorname{Co}(S(\mu)) \backslash\left(S(\mu) \cup\left[b-a^{\prime}, b+a^{\prime}\right]\right)$ contains exactly one zero of $p_{n}$ which lies beyond a prescribed sufficiently small distance from $K$. Using this, the upper bound in (16) on $K$ is immediate on account of (14). Thus we have that the family $\left\{p_{n} g_{n}\right\}$ is uniformly bounded on each compact subset of $\mathbb{C} \backslash\left(S(\mu) \cup\left[b-a^{\prime}, b+a^{\prime}\right]\right)$. Suppose that on the compact set $K$ we had chosen before, $\left|p_{n} g_{n}\right|$ is not uniformly bounded 
from below by a positive constant. Take a convergent subsequence of $\left\{p_{n} g_{n}\right\}$ whose limit function has a zero at $z_{0} \in K$. The limit function cannot be identically equal to zero because that would contradict the lower bound which was shown to be true on compact subsets of the complement of the convex hull of the support. Therefore, $z_{0}$ is an isolated zero. Choose a neighborhood $V$ of $z_{0}$ at a positive distance from $S(\mu) \cup\left[b-a^{\prime}, b+a^{\prime}\right]$. By Hurwitz Theorem, we conclude that there is a subsequence of indices $\Delta$ such that for each $n \in \Delta, p_{n} g_{n}$ has at least one zero in $V$. Such zeros must be contained in the real line since as was proved earlier $p_{n} g_{n}$ does not have zeros outside the real line for any $n$. Let us show that they cannot be on the real line either for all sufficiently large $n$. Having proved this we would arrive to a contradiction which implies that on $K$ the sequence $\left\{\left|p_{n} g_{n}\right|\right\}$ is uniformly bounded from below on $K$ by a positive constant as needed.

First of all, using the arguments employed above we know that for all sufficiently large $n$ we can guarantee that $p_{n}$ has no zero on $V$. Let us prove that for all sufficiently large $n, g_{n}$ does not have any zero on $\operatorname{Co}(S(\mu)) \backslash\left[b-a^{\prime}, b+a^{\prime}\right]$ and thus on $\mathbb{C} \backslash\left[b-a^{\prime}, b+a^{\prime}\right]$. To this end, notice that

$$
\left(p_{n} g_{n}\right)^{\prime}(z)=\int \frac{p_{n}^{2}(x)}{z-x} d \mu(x)<0, \quad z \in \mathbb{R} \backslash S(\mu) .
$$

Therefore the funcion $p_{n} g_{n}$ has at most one simple zero in each one of the open intervals which give the connected components of $\operatorname{Co}(S(\mu)) \backslash S(\mu)$. On those intervals, we saw that $p_{n}$ has exactly one zero for all sufficiently large $n$; therefore, for such $n$ the functions $g_{n}$ cannot have any zeros. With this we conclude the proof.

Now, let us obtain some integral expression connecting the Stieltjes polynomials and the second kind functions.

Lemma 2. We have

$$
s_{n}(z)-\frac{1}{g_{n-1}(z)}=\frac{1}{g_{n-1}(z)} \int \frac{s_{n}(x)}{z-x} p_{n-1}(x) d \mu(x), \quad z \in \overline{\mathbb{C}} \backslash \operatorname{Co}(S(\mu)),
$$

and

$$
s_{n}(z) g_{n-1}(z)=1+\frac{g_{n-1}(z)}{2 \pi i} \int_{\gamma} \frac{d \zeta}{g_{n-1}(\zeta)(\zeta-z)}
$$

where $\gamma$ is any positively oriented close smooth curve which surrounds $\operatorname{Co}(S(\mu))$ such that $z$ is contained in the unbounded component of $\overline{\mathbb{C}} \backslash \gamma$. If $S(\mu)=\operatorname{ess}[b-a, b+a]$, then we can take $\gamma$ in (19) as any smooth contour surrounding $[b-a, b+a]$ and the formula remains valid for all sufficiently large $n$.

Proof. From the orthogonality relations of $p_{n-1}$ with respect to the measure $\mu$, we obtain

$$
\begin{gathered}
\int \frac{S_{n}(z)-S_{n}(x)}{z-x} p_{n-1}(x) d \mu(x)=\int \frac{z^{n}-x^{n}}{z-x} p_{n-1}(x) d \mu(x) \\
=\int x^{n-1} p_{n-1}(z) d \mu(x)=\frac{1}{\kappa_{n-1}} .
\end{gathered}
$$


Rewriting this equality, we find that

$$
g_{n-1}(z) s_{n}(z)=1+\int \frac{s_{n}(x)}{z-x} p_{n-1}(x) d \mu(x),
$$

which is equivalent to the first formula of the lemma. From (18), (14), and (15) (used with $\ell_{n}(x)=x p_{n-1}(x)$ ), it follows that

$$
s_{n}(z)-\frac{1}{g_{n-1}(z)}=\left(\int \frac{p_{n-1}^{2}(x)}{1-x / z} d \mu(x)\right)^{-1} \int \frac{x s_{n}(x)}{z-x} p_{n-1}^{2}(x) d \mu(x) .
$$

Therefore, this function is analytic in $\overline{\mathbb{C}} \backslash \operatorname{Co}(S(\mu))$ and has a zero of order at least 1 at infinity. Using Cauchy's integral formula with a curve $\gamma$ as indicated above, we obtain

$$
s_{n}(z)-\frac{1}{g_{n-1}(z)}=\frac{1}{2 \pi i} \int_{\gamma} \frac{s_{n}(\zeta)-1 / g_{n-1}(\zeta)}{(z-\zeta)} d \zeta=\frac{1}{2 \pi i} \int_{\gamma} \frac{d \zeta}{g_{n-1}(\zeta)(\zeta-z)},
$$

which is (19) in different form. When $S(\mu)=\operatorname{ess}[b-a, b+a]$, take any smooth contour that surrounds $[b-a, b+a]$. Choose $a^{\prime}>a$ such that $\left[b-a^{\prime}, b+a^{\prime}\right]$ does not intersect the contour. According to Lemma 1 , for all sufficiently large $n, g_{n}$ does not have zeros on $\mathbb{C} \backslash\left[b-a^{\prime}, b+a^{\prime}\right]$. Therefore, reasoning as before we can obtain (19) using this $\gamma$. The proof is complete.

In his letter to Hermite [22], Stieltjes considers the function $g_{n}$ of second kind with respect to the Legendre weight. He notices that such a function has a zero at infinity of degree $n+1$ and concludes that

$$
\frac{1}{g_{n}(z)}=E_{n+1}+\frac{a_{1}}{z}+\frac{a_{2}}{z^{2}}+\cdots, \quad n \in \mathbb{N},
$$

where $E_{n+1}$ is a polynomial of degree $n+1$. Using Cauchy's integral formula he obtains an integral representation of the polynomial $E_{n+1}$ (different from (18) and (19)) which allows him to prove that it satisfies full orthogonality relations with respect to $p_{n}(x) d x$. Therefore, $E_{n+1}$ is $S_{n+1}$ up to a multiplicative constant. We have preferred to take (2) as the starting-point for the Stieltjes polynomials.

\section{Asymptotics of Stieltues Polynomials}

Recall that

$$
E=\left\{z \in \mathbb{C}: g_{\Omega}(z, \infty) \leq \max _{w \in \operatorname{Co}(S(\mu))} g_{\Omega}(w, \infty)\right\} .
$$

The set $E$ contains the convex hull of $S(\mu)$ and has, in general, non empty interior. Moreover, $E$ coincides with $S(\mu)$ (and thus has empty interior) if and only if $S(\mu)$ is connected (an interval). It is well known that the Green function $g_{\Omega}(z, \infty)$ tends to zero, except at most on a set of capacity zero, as $z$ goes to $S(\mu)$.

Let $f$ be a bounded function defined on $K$. As usual, $\|f\|_{K}=\sup \{|f(z)|: z \in K\}$. The following theorem provides a stronger version of (10). 
Theorem 2. Let $\mu \in \mathbf{R e g}$ and $\operatorname{cap} S(\mu)>0$. Then

$$
\limsup _{n \rightarrow \infty}\left\|s_{n+1} g_{n}-1\right\|_{K}^{1 / n} \leq\left\|\exp \left\{-g_{\Omega}(\cdot, \infty)\right\}\right\|_{K}\left\|\exp \left\{g_{\Omega}(\cdot, \infty)\right\}\right\|_{\operatorname{Co}(S(\mu))},
$$

where $K$ is any compact subset of $\overline{\mathbb{C}} \backslash E$. Moreover, if additionally we suppose that $S(\mu)=$ $\operatorname{ess}[b-a, b+a]$, then

$$
\limsup _{n \rightarrow \infty}\left\|s_{n+1} g_{n}-1\right\|_{K}^{1 / n} \leq\left\|\exp \left\{-g_{\Omega}(\cdot, \infty)\right\}\right\|_{K},
$$

with $K$ any compact subset of $\overline{\mathbb{C}} \backslash S(\mu)$.

Proof. Fix a compact set $K \subset \mathbb{C} \backslash E$. Let $r$ be a real number, $r>\left\|g_{\Omega}(\cdot, \infty)\right\|_{\mathrm{Co}(S(\mu))}$, such that $K$ lies in the unbounded component of $\mathbb{C} \backslash \gamma_{r}$, where $\gamma_{r}=\left\{\zeta \in \mathbb{C}: g_{\Omega}(\zeta, \infty)=r\right\}$. Obviously, $\gamma_{r}$ surrounds $\operatorname{Co}(S(\mu))$. From (19), applied integrating over $\gamma_{r}$, we have that

$$
\left\|s_{n+1} g_{n}-1\right\|_{K} \leq C \frac{\left\|g_{n}\right\|_{K}}{\inf _{\zeta \in \gamma_{r}}\left|g_{n}(\zeta)\right|},
$$

where $C$ is a positive constant depending on the length of $\gamma_{r}$ and the distance between $\gamma_{r}$ and $K$. Therefore,

$$
\limsup _{n \rightarrow \infty}\left\|s_{n+1} g_{n}-1\right\|_{K}^{1 / n} \leq \frac{\limsup _{n \rightarrow \infty}\left\|g_{n}\right\|_{K}^{1 / n}}{\lim _{n \rightarrow \infty} \inf _{\zeta \in \gamma_{r}}\left|g_{n}(\zeta)\right|^{1 / n}} .
$$

By (16) and (5), we have that

$$
\lim _{n \rightarrow \infty}\left|g_{n}(z)\right|^{1 / n}=\exp \left\{-g_{\Omega}(z, \infty)\right\},
$$

uniformly on compact subsets of $\mathbb{C} \backslash \operatorname{Co}(S(\mu))$. From (23), it follows that

$$
\limsup _{n \rightarrow \infty}\left\|g_{n}\right\|_{K}^{1 / n}=\left\|\exp \left\{-g_{\Omega}(\cdot, \infty)\right\}\right\|_{K}
$$

and

$$
\lim _{n \rightarrow \infty} \inf _{\zeta \in \gamma_{r}}\left|g_{n}(\zeta)\right|^{1 / n}=\exp \{-r\} .
$$

Relations (24) and (25) together with (22) give

$$
\limsup _{n \rightarrow \infty}\left\|s_{n+1} g_{n}-1\right\|_{K}^{1 / n} \leq \exp \{r\}\left\|\exp \left\{-g_{\Omega}(\cdot, \infty)\right\}\right\|_{K} .
$$

The left hand of this inequality does not depend on $r$; therefore, we can make $r$ tend to $\left\|g_{\Omega}(\cdot, \infty)\right\|_{\mathrm{Co}(S(\mu))}$ obtaining (20) for compact subsets of $\mathbb{C} \backslash E$. The function under the norm sign on the left hand of $(20)$ is analytic on $\overline{\mathbb{C}} \backslash S(\mu)$ and, in particular, on $\overline{\mathbb{C}} \backslash E$; therefore, by use of the Maximum Principle the result is easily extended to compact subsets of $\overline{\mathbb{C}} \backslash E$.

The proof of (21) is analogous to that of (20) taking advantage of the special structure of $S(\mu)$. To avoid repetitions we simply outline the main ingredients. We start out again with a fixed compact subset $K$ which is now contained in $\mathbb{C} \backslash S(\mu)$. Take $r>0$ sufficiently small so that $K$ lies entirely on the unbounded component of the complement of $\gamma_{r}$, taking care that $\gamma_{r}$ does not intersect any of the mass points which $S(\mu)$ has outside of $[b-a, b+a]$. According to Lemma 1 we know that for all sufficiently large $n, g_{n}$ has no zero on or outside $\gamma_{r}$. Therefore, according to Lemma 2, (19) remains valid integrating over this $\gamma_{r}$ and we 
deduce a bound analogous to (22). Now, (5) takes place uniformly on compact subsets of $\mathbb{C} \backslash S(\mu)$ (see Theorem 3.1.1 and Corollary 1.1.5 in [21]) taking into consideration that the zeros of $p_{n}$ are bounded away from $K$. Using this and (16), we obtain (23) on each compact subset of $\mathbb{C} \backslash S(\mu)$ and we can proceed as before, with the advantage that we can make $r$ approach zero. With this we conclude the proof.

With the aid of this theorem, we are able to prove our main result stated in the introduction.

Proof of Theorem 1. a) Since $\left\|\exp \left\{-g_{\Omega}(z, \infty)\right\}\right\|_{K}\left\|\exp \left\{g_{\Omega}(z, \infty)\right\}\right\|_{\operatorname{Co}(S(\mu))}<1$ due to the harmonicity of $g_{\Omega}(z, \infty)$ on $\overline{\mathbb{C}} \backslash E$, relation (10) follows immediately from (20). The statement concerning the zeros of $\left\{S_{n+1}\right\}_{n \in \mathbb{N}}$ is a direct consequence of (10) and Hurwitz' Theorem since the function 1 has no zeros on $\overline{\mathbb{C}} \backslash E$. Finally, (10) and (23) render (11). The case when $S(\mu)=\operatorname{ess}[b-a, b+a]$ is proved analogously using (21) in place of (20).

b) First, notice that it is sufficient to consider the case when $a=1$ and $b=0$ : the general case may be reduced to this one by means of an affine change of variables. Secondly, according to (21), under the present conditions we know that (10) takes place uniformly on each compact subset of $\overline{\mathbb{C}} \backslash S(\mu)$. Finally, from (14) and (7), we have that

$$
\lim _{n \rightarrow \infty} g_{n}(z) p_{n}(z)=\frac{1}{\pi} \int_{-1}^{1} \frac{d x}{(z-x) \sqrt{1-x^{2}}}=\frac{1}{\sqrt{z^{2}-1}},
$$

uniformly on each compact subset of $\overline{\mathbb{C}} \backslash S(\mu)$. Putting these things together and using (6), we obtain

$$
\begin{gathered}
\lim _{n \rightarrow \infty} \frac{s_{n+1}(z)}{s_{n}(z)}=\lim _{n \rightarrow \infty} \frac{s_{n+1}(z) g_{n}(z)}{s_{n}(z) g_{n-1}(z)} \times \\
\lim _{n \rightarrow \infty} \frac{p_{n-1}(z) g_{n-1}(z)}{p_{n}(z) g_{n}(z)} \times \lim _{n \rightarrow \infty} \frac{p_{n}(z)}{p_{n-1}(z)}=z+\sqrt{z^{2}-1},
\end{gathered}
$$

and all the limits hold uniformly on each compact subset $K$ of $\mathbb{C} \backslash S(\mu)$. With this we prove the first part of (12). From (26) and (10), we immediately obtain the second relation.

c) From (10), (8), and (26), we have

$$
\begin{aligned}
\lim _{n \rightarrow \infty} \frac{s_{n+1}(z)}{[\Psi(z)]^{n}} & =\lim _{n \rightarrow \infty} s_{n+1}(z) g_{n}(z) \times \lim _{n \rightarrow \infty} \frac{p_{n}(z)}{[\Psi(z)]^{n}} \\
& \times \lim _{n \rightarrow \infty} \frac{1}{g_{n}(z) p_{n}(z)}=\sqrt{\frac{z^{2}-1}{2 \pi}} S_{\mu}(\Psi(z)),
\end{aligned}
$$

with uniform convergence on any compact subset $K$ of $\mathbb{C} \backslash[-1,1]$, which proves (13).

Comparing these results with (5), (6), and (8), one observes that, for these important classes of measures, there are points in common between the asymptotic behaviour of Stieltjes polynomials and of orthonormal polynomials; this is specially so when $S(\mu)=$ $\operatorname{ess}[b-a, b+a]$. When the support of the measure already contains two whole intervals some differences do arise as the example in Section 6 illustrates. That example also reveals that Theorem 2 is, in some sense, sharp. 
With respect to the location of the zeros, it is known (cf. [14] and [18]) that, in general, Stieltjes polynomials may have complex zeros. Despite this fact, statement a) of Theorem 1 shows that the zeros may only accumulate on $E$ (on $S(\mu)$ if $S(\mu)=\operatorname{ess}[b-a, b+a]$ ) when $\mu \in \mathbf{R e g}$. We will complement this assertion in the next result. In order to state it properly it is necessary to give some additional definitions. It is well known (see [20], Section 3.3) that among all probability measures $\sigma$ with support in $S(\mu)$ there exists a probability measure $\lambda$ (which is unique if cap $S(\mu)>0$ ) with support in $S(\mu)$, called the extremal or equilibrium measure of $S(\mu)$, minimizing the energy

$$
\mathcal{I}(\sigma)=\iint \log \frac{1}{|z-t|} d \sigma(z) d \sigma(t)
$$

Let $P(\sigma ; z)=-\int \log |z-t| d \sigma(t)$ be the potential of the measure $\sigma$. There exists a constant $F$, called the equilibrium constant of $S(\mu)$, such that

$$
\begin{aligned}
& P(\lambda ; z) \leq F, \quad z \in \mathbb{C}, \\
& P(\lambda ; z)=F, \quad z \in S(\mu) \backslash A \quad \text { with } \quad \operatorname{cap} A=0 .
\end{aligned}
$$

It may be proved that the property above characterizes the equilibrium measure and that the equilibrium constant $\mathrm{F}$ is precisely the minimal energy $\mathcal{I}(\lambda)$. We also remind that cap $S(\mu)=\exp \{-F\}$. If cap $S(\mu)>0$, the equilibrium measure of $S(\mu)$ is closely related to the Green function relative to the region $\overline{\mathbb{C}} \backslash S(\mu)$ by means of the formula

$$
g_{\Omega}(z, \infty)=F-P(\lambda ; z), \quad z \in \mathbb{C} \backslash S(\mu) .
$$

Let $\rho_{n}$ and $\rho$ be finite Borel measures on $\overline{\mathbb{C}}$. By $\rho_{n} \stackrel{*}{\longrightarrow} \rho, n \rightarrow \infty$, we denote the weak* convergence of $\rho_{n}$ to $\rho$ as $n$ tends to infinity. This means that for every continuous function $f$ on $\overline{\mathbb{C}}$

$$
\lim _{n \rightarrow \infty} \int f(x) d \rho_{n}(x)=\int f(x) d \rho(x)
$$

For a given polynomial $T$, we denote by $\Lambda_{T}$ the normalized zero counting measure of $T$. That is

$$
\Lambda_{T}=\frac{1}{\operatorname{deg} T} \sum_{\xi: T(\xi)=0} \delta_{\xi}
$$

The sum is taken over all the zeros of $T$ and $\delta_{\xi}$ denotes the Dirac measure concentrated at $\xi$.

Theorem 3. Suppose that $S(\mu)=\operatorname{ess}[b-a, b+a], a>0$, and $\mu \in \mathbf{R e g}$. Then

$$
\Lambda_{S_{n+1}} \stackrel{*}{\longrightarrow} \frac{d x}{\pi \sqrt{a^{2}-(x-b)^{2}}}, \quad n \rightarrow \infty .
$$

Proof. Set $\Lambda_{S_{n+1}} \equiv \Lambda_{n}$. In this case it is known (see [20], Corollary 5.2.4) that $\operatorname{cap} S(\mu)=a / 2$, that $g_{\Omega}(z, \infty) \equiv \log |\Psi((z-b) / a)|$, and the equilibrium measure $\lambda$ is $d x /\left(\pi \sqrt{a^{2}-(x-b)^{2}}\right)$.

All the measures $\Lambda_{n}$ are probability measures. Let $\Delta \subset \mathbb{N}$ be a subsequence of indices such that

$$
\Lambda_{n} \stackrel{*}{\longrightarrow} \Lambda, \quad n \in \Delta, \quad n \rightarrow \infty .
$$


It is sufficient to prove that $\Lambda \equiv \lambda$ for any such sequence $\Delta$ of indices. According to Theorem 1, in this case the support of $\Lambda$ is contained in the set $S(\mu)$. Taking (11), (27), and (4) into account, we have that

$$
\lim _{n \in \Delta} P\left(\Lambda_{n} ; z\right)=\lim _{n \in \Delta} \frac{-1}{n+1} \log \left|S_{n+1}\right|=P(\lambda ; z), \quad z \in \mathbb{C} \backslash S(\mu) .
$$

On the other hand, from (28) one obtains

$$
\lim _{n \in \Delta} P\left(\Lambda_{n} ; z\right)=P(\Lambda ; z), \quad z \in \mathbb{C} \backslash S(\mu)
$$

Thus, $P(\Lambda ; z)=P(\lambda ; z)$ except at most on a set of Lebesgue measure zero in the complex plane; therefore, from Theorem 3.7.4 in [20], we obtain that $\Lambda \equiv \lambda$ as we wanted to prove.

Using basically the same arguments, one can show that the balayage onto $\partial E$ of any convergent subsequence of $\left\{\Lambda_{S_{n+1}}\right\}_{n \in \mathbb{N}}$ is the balayage onto $\partial E$ of the corresponding equilibrium measure $\lambda$.

\section{Padé-type Approximation}

The first part of Theorem 1 may be applied to estimate the rate of convergence of a certain sequence of interpolating rational functions to Markov functions when part of the poles are fixed at the zeros of the orthogonal polynomials of the given measure. Set

$$
\widehat{\mu}(z)=c+\int \frac{d \mu(x)}{z-x}, \quad z \in \overline{\mathbb{C}} \backslash S(\mu), \quad c \in \mathbb{R} .
$$

Let $p_{n}$ be the $n$th orthonormal polynomial with respect to $\mu$. It is easy to verify that there exists a unique rational function $R_{n}=L_{n} /\left(Q_{n} p_{n}\right)$, such that $L_{n}$ and $Q_{n}$ satisfy:

- $\operatorname{deg} Q_{n} \leq n+1, \operatorname{deg} L_{n} \leq 2 n+1$, and $Q_{n} \not \equiv 0$.

- $Q_{n}(z) p_{n}(z) \widehat{\mu}(z)-L_{n}(z)=\mathcal{O}\left(\frac{1}{z^{n+2}}\right), \quad z \rightarrow \infty$.

From the definition it follows immediately, using the Cauchy and Fubini Theorems, that $Q_{n}$ satisfies

$$
\int x^{k} Q_{n}(x) p_{n}(x) d \mu(x)=0, \quad k=0,1, \ldots, n .
$$

Therefore, taking $Q_{n}$ to be monic we have that $Q_{n}=S_{n+1}$. Another immediate consequence of the definition and Cauchy's integral formula (taking into account that $Q_{n}=$ $\left.S_{n+1}\right)$ is

$$
\widehat{\mu}(z)-R_{n}(z)=\frac{1}{\left(s_{n+1}^{2} p_{n}\right)(z)} \int \frac{\left(s_{n+1}^{2} p_{n}\right)(x)}{z-x} d \mu(x), \quad z \in \overline{\mathbb{C}} \backslash S(\mu) .
$$

Using the remainder formula above and the $n$th root asymptotic behaviour of the polynomials $p_{n}$ and $s_{n+1}$, we can prove

Theorem 4. Let $\mu \in \mathbf{R e g}$ and cap $S(\mu)>0$. Then, on each compact subset $K$ of $\overline{\mathbb{C}} \backslash E$, we have

$$
\limsup _{n \rightarrow \infty}\left\|\widehat{\mu}(z)-R_{n}(z)\right\|_{K}^{1 / 3 n} \leq\left\|\exp \left\{-g_{\Omega}(\cdot, \infty)\right\}\right\|_{K}\left\|\exp \left\{g_{\Omega}(\cdot, \infty)\right\}\right\|_{\operatorname{Co}(S(\mu))} .
$$


If, additionally, we suppose that $S(\mu)=[b-a, b+a], a>0$, then

$$
\limsup _{n \rightarrow \infty}\left\|\widehat{\mu}(z)-R_{n}(z)\right\|_{K}^{1 / 3 n} \leq\left\|\exp \left\{-g_{\Omega}(\cdot, \infty)\right\}\right\|_{K}
$$

where $K$ is any compact subset of $\overline{\mathbb{C}} \backslash S(\mu)$.

Proof. Fix a compact set $K \subset \mathbb{C} \backslash E$. Let $r$ be a real number, $r>\left\|g_{\Omega}(\cdot, \infty)\right\|_{\mathrm{Co}(S(\mu))}$, such that $K$ lies in the unbounded component of $\mathbb{C} \backslash \gamma_{r}$, where $\gamma_{r}=\left\{\zeta \in \mathbb{C}: g_{\Omega}(\zeta, \infty)=r\right\}$. For short, let us denote $1 /\left(s_{n+1}^{2}(z) p_{n}(z)\right)$ by $h_{n}(z)$. From $(29)$, we have that

$$
\widehat{\mu}(z)-R_{n}(z)=h_{n}(z) \int \frac{1}{h_{n}(x)} \frac{d \mu(x)}{z-x}, \quad z \in K
$$

Since for each $z \in K, 1 /\left((z-x) h_{n}(x)\right)$ is analytic in an open neighbourhood of the bounded component of $\mathbb{C} \backslash \gamma_{r}$, we may use Cauchy's integral formula to obtain

$$
\begin{aligned}
\widehat{\mu}(z)-R_{n}(z) & =h_{n}(z) \int\left(\frac{1}{2 \pi i} \int_{\gamma_{r}} \frac{1}{h_{n}(\zeta)(\zeta-x)} \frac{d \zeta}{z-\zeta}\right) d \mu(x) \\
& =\frac{h_{n}(z)}{2 \pi i} \int_{\gamma_{r}}\left(\int \frac{d \mu(x)}{\zeta-x}\right) \frac{d \zeta}{h_{n}(\zeta)(z-\zeta)}=\frac{h_{n}(z)}{2 \pi i} \int_{\gamma_{r}} \frac{1}{h_{n}(\zeta)} \frac{\widehat{\mu}(\zeta)}{z-\zeta} d \zeta,
\end{aligned}
$$

where Fubini's Theorem has been used in the second equality. Hence

$$
\left\|\widehat{\mu}(z)-R_{n}(z)\right\|_{K} \leq C \frac{\left\|h_{n}\right\|_{K}}{\inf _{\zeta \in \gamma_{r}}\left|h_{n}(\zeta)\right|},
$$

where $C$ is a positive constant depending on the length of $\gamma_{r}$ and the distance between $\gamma_{r}$ and $K$. Therefore,

$$
\limsup _{n \rightarrow \infty}\left\|\widehat{\mu}(z)-R_{n}(z)\right\|_{K}^{1 / 3 n} \leq \frac{\limsup _{n \rightarrow \infty}\left\|h_{n}\right\|_{K}^{1 / 3 n}}{\lim _{n \rightarrow \infty} \inf _{\zeta \in \gamma_{r}}\left|h_{n}(\zeta)\right|^{1 / 3 n}} .
$$

From (11) and (5), we obtain

$$
\lim _{n \rightarrow \infty}\left|h_{n}(z)\right|^{1 / 3 n}=\exp \left\{-g_{\Omega}(z, \infty)\right\}
$$

uniformly on compact subsets of $\mathbb{C} \backslash E$. By use of (33), we obtain

$$
\limsup _{n \rightarrow \infty}\left\|h_{n}\right\|_{K}^{1 / 3 n}=\left\|\exp \left\{-g_{\Omega}(z, \infty)\right\}\right\|_{K} \quad \text { and } \quad \lim _{n \rightarrow \infty} \inf _{\zeta \in \gamma_{r}}\left|h_{n}(\zeta)\right|^{1 / 3 n}=\exp \{-r\} .
$$

Relations (34) together with (32) give

$$
\limsup _{n \rightarrow \infty}\left\|\widehat{\mu}(z)-R_{n}(z)\right\|_{K}^{1 / 3 n} \leq \exp \{r\}\left\|\exp \left\{-g_{\Omega}(z, \infty)\right\}\right\|_{K}
$$

The left hand of this inequality does not depend on $r$; therefore, we can make $r$ tend to $\left\|g_{\Omega}(\cdot, \infty)\right\|_{\operatorname{Co}(S(\mu))}$ obtaining (30) for compact subsets of $\mathbb{C} \backslash E$. Since the function under the norm on the left hand of (30) is analytic on a neighbourhood of infinity, from the Maximum Principle it is obvious that (30) is also true for any $K \subset \overline{\mathbb{C}} \backslash E$. Formula (31) is a direct consequence of (30) when the support is an interval.

So far, most papers dealing with Padé-type approximants of Markov type functions, take the distinct fixed poles to have even order (cf. [2] and [3]). This is done in order to 
ensure that the polynomials, whose zeros are the free poles of the rational approximant, be orthogonal with respect to a positive measure. This simplifies matters quite a bit because it forces the free poles to fall on the convex hull of the support of the measure. The question arises whether this restriction, due to the method used in the proofs, may be dropped or weakened. Theorem 4 is a first step in that direction.

\section{Gauss-Kronrod quadrature}

We first introduce an extended Gauss-Kronrod quadrature formula. Let us consider the partial fraction decomposition of the approximant $R_{n}$

$$
R_{n}(z)=\sum_{i=1}^{N} \sum_{j=0}^{M_{i}} \frac{j ! a_{i, j, n}}{\left(z-z_{n, i}\right)^{j+1}} .
$$

$N$ denotes the total number of distinct poles of $R_{n}$. The points $z_{n, i}$ are the zeros of $s_{n+1} p_{n}$. Though the zeros of $p_{n}$ are simple they may coincide with zeros of $s_{n+1}$; therefore, for given $z_{n, i}$ any value of $M_{i}$ is possible (of course $M_{i} \leq n+1$ ). Obviously, $N=N(n)$ and $M_{i}=M_{i}(n)$, but in order to simplify the notation, we omit the explicit reference to this dependence.

Let $f$ be an analytic function on a neighbourhood $V$ of the compact set $E$. Set

$$
I(f)=\int f(x) d \mu(x), \quad I_{n}(f)=\sum_{i=1}^{N} \sum_{j=0}^{M_{i}} a_{i, j, n} f^{(j)}\left(z_{n, i}\right), \quad E_{n}(f)=I(f)-I_{n}(f) .
$$

If $\mu \in \mathbf{R e g}$ and cap $S(\mu)>0$, from a) of Theorem 1 , we know that for $n \geq n_{0}(V)$ all the zeros of $s_{n+1}$ are contained in $V$ and the expressions above make sense. In the sequel, we only consider sufficiently large $n$ 's. Notice that if the zeros $z_{n, i}$ are all simple (which is not known in general), then $I_{n}$ is the usual Gauss-Kronrod quadrature formula. This fact is made explicit by the following lemma where we study the degree of exactness of the quadrature formula just introduced in the space of polynomials.

Lemma 3. There exists $N \in \mathbb{N}$ such that for each $n \geq N$ we have

$$
I(h)=I_{n}(h),
$$

where $h$ is any polynomial of degree less than or equal to $3 n+1$.

Proof. Let $V$ be a neighbourhood of $E$. Let $\gamma$ be an analytic Jordan curve such that $V$ lies in the bounded component of $\mathbb{C} \backslash \gamma$. For $n \geq N$, all the zeros of $S_{n+1}$ belong to $V$ and, therefore, $\widehat{\mu}-R_{n}$ is holomorphic in $\overline{\mathbb{C}} \backslash \bar{V}$. From (29) we know that

$$
\widehat{\mu}(z)-R_{n}(z)=\mathcal{O}\left(\frac{1}{z^{3 n+3}}\right), \quad z \rightarrow \infty .
$$

Then, if $h$ is any polynomial of degree less than or equal to $3 n+1, h\left(\widehat{\mu}-R_{n}\right)$ has a zero at infinity of multiplicity at least two. Therefore, we can use Cauchy's Theorem, Fubini's Theorem, and Cauchy's integral formula to obtain, for $n \geq N$

$$
\begin{aligned}
0 & =\int_{\gamma} h(\zeta)\left(\widehat{\mu}-R_{n}\right)(\zeta) d \zeta=\int_{\gamma} h(\zeta)\left(\int \frac{d \mu(x)}{\zeta-x}\right) d \zeta-\sum_{i=1}^{N} \sum_{j=0}^{M_{i}} a_{i, j, n} j ! \int_{\gamma} \frac{h(\zeta)}{\zeta-z_{n, i}} d \zeta \\
& =\int\left(\int_{\gamma} \frac{h(\zeta)}{\zeta-x} d \zeta\right) d \mu(x)-2 \pi i \sum_{i=1}^{N} \sum_{j=0}^{M_{i}} a_{i, j, n} h^{(j)}\left(z_{n, i}\right)=2 \pi i\left[I(h)-I_{n}(h)\right] .
\end{aligned}
$$


Finally, we estimate the error of this extended Gauss-Kronrod quadrature formula for analytic functions. In the following statement, $E_{n}(f)$ should be understood in the sense of (35). In case that all the zeros of $S_{n+1} p_{n}$ are simple it coincides with (3).

Theorem 5. Let $f$ be an analytic function on a neighbourhood $V$ of $E$. Let $\mu \in \mathbf{R e g}$ and $\operatorname{cap} S(\mu)>0$. Then, we have

$$
\limsup _{n \rightarrow \infty}\left|E_{n}(f)\right|^{1 / 3 n} \leq\left\|\exp \left\{-g_{\Omega}(\cdot, \infty)\right\}\right\|_{\partial V}\left\|\exp \left\{g_{\Omega}(\cdot, \infty)\right\}\right\|_{\operatorname{Co}(S(\mu))},
$$

where $\partial V$ represents the set of boundary points of $V$. If $S(\mu)=[b-a, b+a]$ then

$$
\limsup _{n \rightarrow \infty}\left|E_{n}(f)\right|^{1 / 3 n} \leq\left\|\exp \left\{-g_{\Omega}(\cdot, \infty)\right\}\right\|_{\partial V},
$$

for any function $f$ analytic on a neighbourhood $V$ of $[b-a, b+a]$.

Proof. Let $W$ be a neighbourhood of $E$ with $\bar{W} \subset V$. There exists a natural number $n_{0}(W)$ such that for each $n \in \mathbb{N}$ with $n \geq n_{0}(W)$ the polynomial $S_{n+1}$ has all its zeros contained in the open set $W$.

Let $\gamma$ be an analytic Jordan curve contained in $V$ such that $W$ lies in the bounded component of $\mathbb{C} \backslash \gamma$. Using the Fubini and Cauchy Theorems, we have

$$
I(f)-I_{n}(f)=\frac{1}{2 \pi i} \int_{\gamma} f(\zeta)\left(\widehat{\mu}-R_{n}\right)(\zeta) d \zeta .
$$

From this equality and (30), we obtain

$$
\limsup _{n \rightarrow \infty}\left|I(f)-I_{n}(f)\right|^{1 / 3 n} \leq\left\|\exp \left\{-g_{\Omega}(\cdot, \infty)\right\}\right\|_{\gamma}\left\|\exp \left\{g_{\Omega}(\cdot, \infty)\right\}\right\|_{\operatorname{Co}(S(\mu))} \cdot
$$

We can choose $\gamma$ as close to $\partial V$ as we want, so (36) immediately follows. Obviously, (37) is a direct consequence of (36).

Notice that under the conditions of Theorem 5 we have that $\lim _{n \rightarrow \infty} E_{n}(f)=0$ with geometric rate of order $3 n$. The closer $\partial V$ is to $E$, the slower $E_{n}(f)$ tends to 0 . We wish to point out that Theorem 5 ensures the convergence of Gauss-Kronrod quadrature formula for analytic functions regardless of the signs which the coefficients $a_{i, j, n}$ may have. This approach allows us to obtain estimates for the rate of convergence of Gauss-Kronrod quadrature formulas for a very general class of measures as compared with the measures considered in [6] and [15]. Also, the order of convergence which we give is better than that which follows from Theorem 1 in [6]. As regards [15], it is more difficult to compare the order of convergence because of the different nature of the estimates.

Finally, we remark that (31) and (37) may also be proved imposing on the support of the measure the weaker condition $S(\mu)=\operatorname{ess}[b-a, b+a]$.

\section{ExAMPLE}

The next example illustrates the nature of the difficulties one encounters in trying to improve the results when $S(\mu)$ contains more than one interval. In fact it shows that, in general, in the class of regular measures one cannot obtain asymptotics on a set larger 
than $\mathbb{C} \backslash E$, or get estimates of the rate of convergence better than that expressed on the right hand of $(20)$.

Recall that $p_{n} g_{n}$ has at most one simple zero in each one of the open intervals which give the connected components of $\mathbb{R} \backslash S(\mu)$ (see (17)).

Set $d \mu(x)=w(x) d x$, where $w(x)$ is an even function defined on $[-\beta,-\alpha] \cup[\alpha, \beta], \beta>$ $\alpha>0$. This measure is symmetric with respect to the origin. Therefore, $p_{2 n+1}$ is an odd function and it must have a zero at $z=0$; thus, according to what was said above, $g_{2 n+1}$ does not have any zero in $(-\alpha, \alpha)$. On the contrary, $p_{2 n}$ is even and thus $p_{2 n} / x$ is odd from which it follows that $g_{2 n}(0)=-\int p_{2 n}(x) / x d \mu(x)=0$.

Let $r>0$ and set $\gamma_{r}=\left\{\zeta \in \mathbb{C}: g_{\Omega}(z, \infty)=r\right\}$. Since $g_{2 n+1}^{-1}$ is analytic in $\mathbb{C} \backslash S(\mu)$, we can prove (19) with $\gamma=\gamma_{r}$ reasoning as in Lemma 2. With this formula on $\gamma_{r}$, following the same proof as in Theorem 2, it is easy to obtain that

$$
\limsup _{n \rightarrow \infty}\left\|s_{2 n+2} g_{2 n+1}-1\right\|_{K}^{1 / 2 n} \leq\left\|\exp \left\{-g_{\Omega}(z, \infty)\right\}\right\|_{K}
$$

on each compact subset $K \subset \overline{\mathbb{C}} \backslash S(\mu)$.

From symmetry, it is not difficult to see that $g_{\Omega}(0, \infty)=\max _{\zeta \in[-\beta, \beta]} g_{\Omega}(\zeta, \infty)$. Take $a \in \mathbb{C} \backslash S(\mu)$, with $g_{\Omega}(a, \infty)<g_{\Omega}(0, \infty)$. Let $0<r<g_{\Omega}(a, \infty)$ and $\gamma$ be a positively oriented circle centered at $z=0$ such that $a, \gamma_{r}$ and $S(\mu)$ lie in the unbounded connected component of the complement of $\gamma$. Since $g_{2 n}^{-1}$ has a simple pole at $z=0$, following the arguments used in proving (19) and using the Residue Theorem one has

$$
\begin{aligned}
& s_{2 n+1}(a) g_{2 n}(a)=1+\frac{g_{2 n}(a)}{2 \pi i} \int_{\gamma_{r}} \frac{d \zeta}{g_{2 n}(\zeta)(\zeta-a)}+\frac{g_{2 n}(a)}{2 \pi i} \int_{\gamma} \frac{d \zeta}{g_{2 n}(\zeta)(\zeta-a)} \\
& =1+\frac{g_{2 n}(a)}{2 \pi i} \int_{\gamma_{r}} \frac{d \zeta}{g_{2 n}(\zeta)(\zeta-a)}+\frac{-g_{2 n}(a)}{a g_{2 n}^{\prime}(0)} .
\end{aligned}
$$

For the integral on the right-hand side it is easy to deduce that

$$
\limsup _{n \rightarrow \infty}\left|\frac{g_{2 n}(a)}{2 \pi i} \int_{\gamma_{r}} \frac{d \zeta}{g_{2 n}(\zeta)(\zeta-a)}\right|^{1 / 2 n} \leq \exp \left\{r-g_{\Omega}(a, \infty)\right\}<1 .
$$

For the third term in (38) (see (17) and take into account that $g_{2 n}(0)=0$ ), we have

$$
\frac{-g_{2 n}(a)}{a g_{2 n}^{\prime}(0)}=\frac{g_{2 n}(a) p_{2 n}(0)}{a \int p_{2 n}^{2}(x) x^{-2} d \mu(x)}
$$

Since

$$
\frac{1}{\beta^{2}} \leq\left|\int \frac{p_{2 n}^{2}(x)}{x^{2}} d \mu(x)\right| \leq \frac{1}{\alpha^{2}}
$$

and $\lim _{n \rightarrow \infty}\left|p_{2 n}(z)\right|^{1 / 2 n}=\exp \left\{g_{\Omega}(z, \infty)\right\}$ uniformly on compact subsets of $\mathbb{C} \backslash S(\mu)$ (because $p_{2 n}$ has no zeros in $\left.(-\alpha, \alpha)\right)$ it follows that

$$
\lim _{n \rightarrow \infty}\left|\frac{g_{2 n}(a)}{a g_{2 n}^{\prime}(0)}\right|^{1 / 2 n}=\exp \left\{g_{\Omega}(0, \infty)-g_{\Omega}(a, \infty)\right\}>1
$$

Therefore, taking account of (38), (39), and (40); we obtain

$$
\lim _{n \rightarrow \infty}\left|s_{2 n+1}(a) g_{2 n}(a)-1\right|^{1 / 2 n}=\exp \left\{g_{\Omega}(0, \infty)-g_{\Omega}(a, \infty)\right\} .
$$

Hence, at point $a, s_{2 n+1}(a) g_{2 n}(a)$ does not converge to 1 . Moreover, it diverges with geometric rate. 


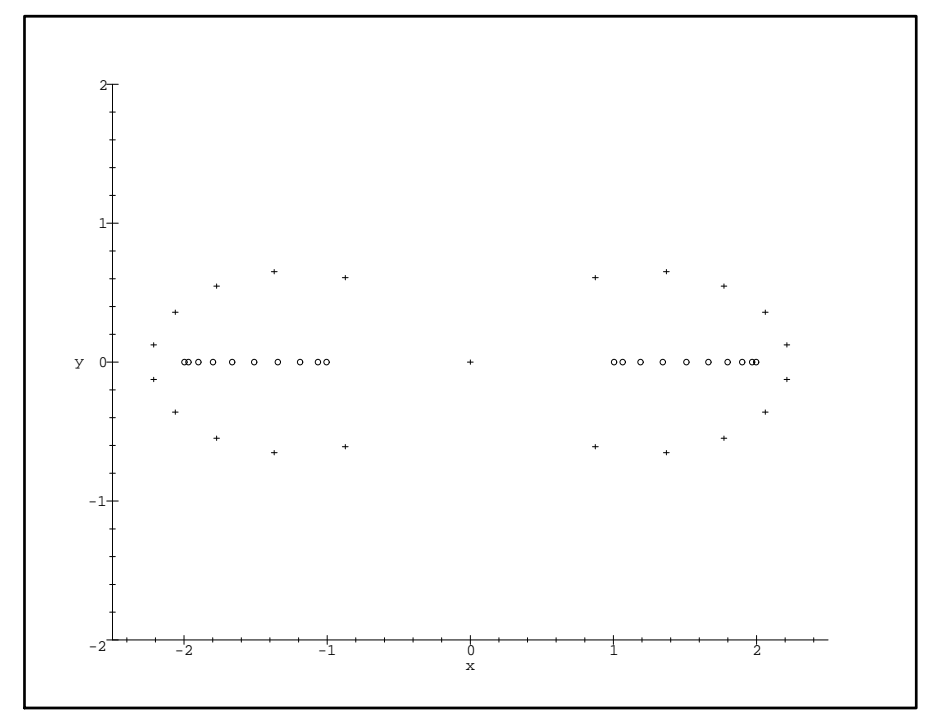

Figure 1 . Zeros of $S_{20}$ and $S_{21}$ for $w \equiv 1, \alpha=1, \beta=2$.

We have considered the particular case $w \equiv 1, \alpha=1, \beta=2$. Numerical experiments show that the zeros of the Stieltjes polynomials for this measure have an interesting behaviour; while the zeros of $S_{2 n}$ sit on $[-2,-1] \cup[1,2]$, those of $S_{2 n+1}$ draw the level curve $\left\{\zeta \in \mathbb{C}: g_{\Omega}(\zeta, \infty)=g_{\Omega}(0, \infty)\right\}$. Figure 1 shows the zeros of $S_{n}$ for $n=20,21$ (the small circles are the zeros of $S_{20}$ and the crosses the zeros of $S_{21}$ ). As this example shows, the only drawback in extending the results of this paper to compact subsets closer to $S(\mu)$ is the existence of zeros of $g_{n}$ in $\operatorname{Co}(S(\mu)) \backslash S(\mu)$. If we know for some reason that the functions of second kind (or some subsequence) have no zeros on $\operatorname{Co}(S(\mu)) \backslash S(\mu)$, then we can extend (20) to any compact subset of $\overline{\mathbb{C}} \backslash S(\mu)$ (for the corresponding subsequence).

\section{REFERENCES}

[1] A. Ambroladze and H. Wallin, Padé-type approximants of Markov and meromorphic functions, J. Approx. Theory 88 (1997), 354-369.

[2] A. Ambroladze and H. Wallin, Extremal polynomials with preassigned zeros and rational approximants, Constr. Approx. 14 (1998), 209-229.

[3] F. Cala and G. López Lagomasino, Multipoint Padé-type approximants. Exact rate of convergence, Constr. Approx. 14 (1998), 259-272.

[4] S. Ehrich, Asymptotic properties of Stieltjes polynomials and Gauss-Kronrod quadrature formulae, J. Approx. Theory 82 (1995), 287-303.

[5] S. EHRICH, Asymptotic behaviour of Stieltjes polynomials for ultraspherical weight functions, $J$. Comput. Appl. Math. 65 (1995), 135-144.

[6] S. Ehrich, Gauss-Kronrod quadrature error estimates for analytic functions, Zeitschr. f. Angew. Mathematik und Mechanik 74 (1995) 6, T691-T693.

[7] S. EHRICH, Stieltjes polynomials and the error of Gauss-Kronrod quadrature formulas, ISNM 131 Proc. Conference Oberwolfach (1999), 57-77.

[8] S. Ehrich and G. Mastroianni, Stieltjes polynomials and Lagrange interpolation, Math. Comp. 66 (1997), 311-331. 
[9] W. Gautschi, Gauss-Kronrod quadrature - a survey, in "Numerical Methods and Approximation Theory III"(G. V. Milovanović, Ed.), 39-66, Niṽ , 1988.

[10] A. S. Kronrod, Nodes and weights for quadrature formulae. Sixteen-Place Tables. Nauka Moscow, 1964, English transl., Consultants Bureau, New York, 1965.

[11] G. Monegato, Positivity of weights of extended Gauss-Legendre quadrature rules, Math. Comp. 32 (1978), 243-245.

[12] G. Monegato, Stieltjes polynomials and related quadrature rules, SIAM Review 24 (1982), 137-158.

[13] P. Nevai, "Orthogonal Polynomials," Memoirs of Amer. Math. Soc. 213, Providence, R.I., 1979.

[14] S. E. Notaris, Some new formulae for the Stieltjes polynomials relative to classical weight functions, SIAM J. Numer. Anal. 28 (1991), 1196-1206.

[15] S. E. Notaris, Error bounds for Gauss-Kronrod quadrature of analytic functions, Numer. Math. 64 (1993), 371-380.

[16] F. Peherstorfer, On the asymptotic behaviour of functions of second kind and Stieltjes polynomials, and on Gauss-Kronrod quadrature formulas, J. Approx. Theory 70 (1992), 156-190.

[17] F. Peherstorfer, Stieltjes polynomials and functions of second kind, J. Comput. Appl. Math. 65 (1995), 319-338.

[18] F. Peherstorfer and K. Petras, Ultraspherical Gauss-Kronrod quadrature is not possible for $\lambda>3$, SIAM J. Numer. Anal. 37 (2000), 927-948.

[19] E. A. Rakhmanov, On asymptotic properties of polynomials orthogonal on the circle with weights not satisfying Szegö's condition, Math. USSR. Sbornik. 58,1 (1987), 149-167.

[20] T. Ransford, "Potential Theory in the Complex Plane," London Mathematical Society, Student Texts 28, Cambridge University Press, New York, 1995.

[21] H. Stahl and V. Totik, "General Orthogonal Polynomials," Cambridge University Press, Cambridge, 1992.

[22] T. J. Stieltues, Correspondence d'Hermite et de Stieltjes, Prentice-Hall, Englewood Cliffs, NJ, 1966.

[23] G. Szegő, "Orthogonal Polynomials," Coll. Publ. XXIII, Amer. Math. Soc., Providence, Rhode Island, 1975 (4th edition).

[24] G. Szegő, Über gewisse orthogonale Polynome, die zu einer oszillierenden Belegungsfunktion gehören, Math. Ann. 110 (1935), 501-513.

M. Bello Hernández, Dpto. de Matemáticas y Computación, Universidad de la Rioja, Edificio J. L. Vives, Luis de Ulloa s/n, 26004 Logroño, Spain

E-mail address: mbello@dmc.unirioja.es

B. de la Calle Ysern, Dpto. de Matemática Aplicada, E. T. S. De Ingenieros Industriales, Universidad Politécnica de Madrid, José G. Abascal 2, 28006 Madrid, Spain

E-mail address: bcalle@etsii.upm.es

J.J. Guadalupe Hernández, Dpto. de Matemáticas y Computación, Universidad de la Rioja, Edificio J. L. Vives, Luis de Ulloa s/n, 26004 Logroño, Spain

E-mail address: joguadal@dmc.unirioja.es

G. López lagomasino, Dpto. de Matemáticas, Escuela Politécnica Superior, Universidad Carlos III de Madrid, Universidad 30, 28911 Leganés, Spain

E-mail address: lago@math.uc3m.es 